\title{
Diffractive Bifocal Contact Lenses in Aphakia and Pseudophakia
}

\section{A Pilot Study}

\author{
K. BARTON, M. H. FREEMAN, E. G. WOODWARD, R. J. BUCKLEY \\ London
}

\begin{abstract}
Summary
Diffractive bifocal contact lenses function by diverting incident light to two focal points. Light from near objects is focused by diffraction while that from distant objects is focused by conventional refraction. Both processes occur simultaneously throughout the full aperture of the lens. Fourteen aphakic and pseudophakic contact lens wearers were fitted with diffractive bifocal lenses and observed for an eight week period. Six $(42.8 \%)$ were satisfied with the visual result. Five subjects $(35.7 \%)$ discontinued lens wear complaining of poor quality of near vision. We conclude that these lenses may help selected aphakic patients to discard their reading glasses but the low acceptance rate suggests that this type of optic is probably not suitable for implantation as an intraocular lens.
\end{abstract}

Since the introduction of the diffractive contact lens, both hard and soft varieties have enjoyed considerable commercial exposure. As a full aperture, simultaneous vision lens the diffractive optic offers a simple method of countering the effects of presbyopia.

Patients with aphakia, either bilaterally or with an accommodating, presbyopic or psedophakic fellow eye comprise a considerable proportion of contact lens practice in Moorfields Eye Hospital. A pilot study was carried out to investigate the potential for correction of both the refractive error in aphakia and the loss of accommodation in aphakia and pseudophakia using diffractive lenses.

Following recent reports using a diffractive intraocular lens, ${ }^{1}$ the predictability of the near visual outcome and the effect on visualisation of the fundus and laser photocoagulation have been questioned. The second part of this report comprises a simple study to investigate the effect of the diffractive optic on examination of the eye in pseudophakia.

\section{Mechanism of action}

The diffractive lens provides distance vision by refraction. Near vision is achieved using. diffraction (Diagrams 1 and 2) from a series of phase-controlled concentric slopes on the posterior lens surface. ${ }^{2,3}$ The narrow intervening steps do not diffract (they scatter light).

The diffractive lens evolved from the diffraction grating (Diagram 3). Rays passing through a slit form secondary wavelets which can interfere with those arriving from another slit to form an image. A diffraction grating of

From: Contact Lens Department, Moorfields Eye Hospital, and The City University, Department of Optometry and Visual Science, London.

Correspondence to K. Barton, Resident Surgical Officer, Moorfields Eye Hospital, City Road, London EC1V 2PD. 


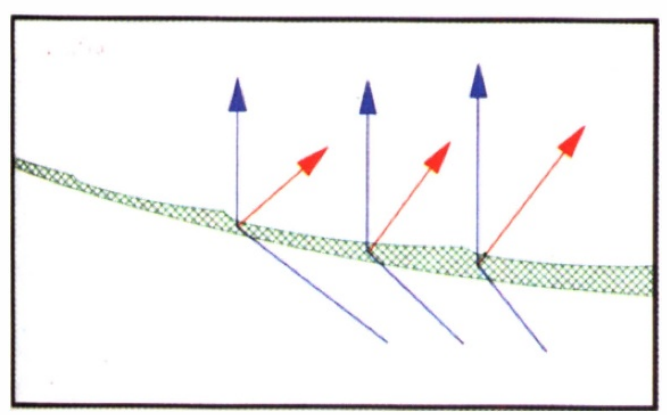

Diagram 1. Cross-section of a diffractive bifocal contact lens. Every point on the lens diffracts and refracts transmitted light. Colour code: Blue and red arrows represent refracted and diffracted rays respectively. Green and black represent transparent and opaque media respectively.

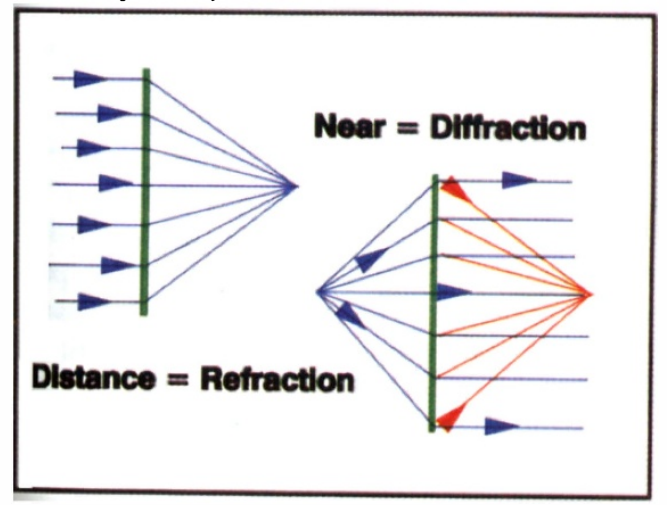

Diagram 2. The diffractive lens focuses light from distant objects on the retina by refraction and near objects by diffraction. Incident light is thus split so that $41.8 \%$ is diffracted to form the near image, $41.8 \%$ refracted to form the distance image and the remainder scattered, or diffracted into other orders.

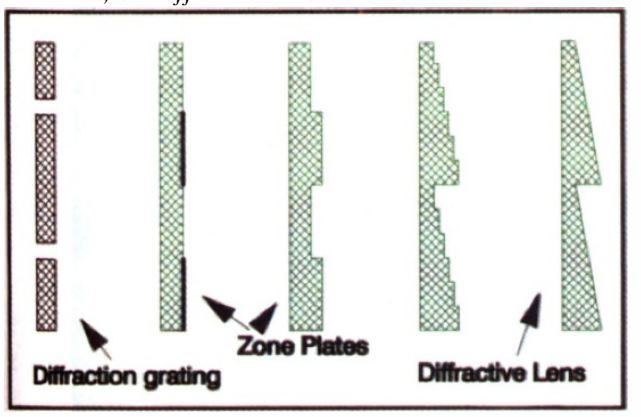

Diagram 3. The diffractive lens evolved via a number of intermediaries from the diffraction grating. The entire lens must transmit and diffract light for an image of adequate intensity to be produced. Diffracted light is manipulated to produce one diffractive image by the sloped posterior lens surface, retarding the arrival of adjacent rays at the focal point by a fraction of a wavelength so they interfere constructively (see 'Mechanism of Action' in text). this type is ineffective as a lens, because it transmits insufficient light, produces multiple images and creates gross chromatic aberration.

Light is not diffracted only at slits, secondary wavelets occur at every point in a beam of light at an interface. The grating therefore evolved to possess alternate transparent and opaque areas (Fresnel's zone plate). In Wood's 'all clear' zone plate alternate half zones differ in thickness by $\frac{1}{2}$ wavelength producing an enhanced image at the near focus by causing light focused at infinity to interfere destructively.

This type of grating would be a better lens than the slit grating but not as good as a blazed grating, which has a smooth phase change across the whole of each zone. This provides more control over the images when made in the form of a lens.

The smooth slope of the diffractive lens divides each annular zone into a staircase of infinitely small steps of phase delay, so that light from every point on the back surface interferes constructively at the focus to produce the near image. The step permits continuity between adjacent zones, behaving as a catching-up zone.

\section{Methods}

1. Fourteen consecutive contact lens wearers were recruited from the contact lens department of Moorfields Eye Hospital to provide a group of patients in each of three categories:

1. Aphakic/Phakic (presbyopic or accommodating)

2. Aphakic/Aphakic

3. Pseudophakic/Phakic with high refractive error or aphakic

Patients were fitted bilaterally (except emmetropic accommodating eyes) with Optoacryl 60 (gas permeable) bifocal contact lenses incorporating distance power with a diffractive addition of +3 dioptres (see table). Recruitment criteria included requirement of a +3 dioptre spectacle addition and no other ocular pathology (macular degeneration, secondary causes of cataract, etc). Patients were assessed over an eight week period of lens wear during which visual acuities, keratometry and corneal pachometry measurements were recorded and general slit lamp 
Table I Details of patients fitted with diffractive bifocal contact lenses

\begin{tabular}{|c|c|c|c|c|c|c|c|c|c|c|c|c|}
\hline No & $\mathrm{R}$ diagnosis & L diagnosis & Outcome & Comment & R Prescription & R Dist & R Near & L Prescription & L Dist & L Near & Age & Sex \\
\hline & \multicolumn{12}{|c|}{ Aphakic/Aphakic } \\
\hline 1 & Aphake & Aphake & Discontinued & $\begin{array}{l}\text { Poor wetting and epithelial } \\
\text { instability }\end{array}$ & $8.2 /+17.00$ & $6 / 6$ & N6 & $8.3 /+18.00$ & $6 / 6$ & N6 & 60 & M \\
\hline 2 & Aphake & Aphake & Successful & & $7.71+13.25$ & 6/9 & N5 & $7.7 /+14.25$ & $6 / 6$ & N5 & 43 & M \\
\hline 3 & Aphake & Aphake & Successful & & $7.6 /+15.50$ & $6 / 9$ & N5 & $7.7 /+15.25$ & $6 / 9$ & N5 & 34 & $\mathrm{~F}$ \\
\hline 4 & Aphake & Aphake & Successful & & $7.8 /+12.25$ & $6 / 6$ & N5 & $7.9 /+13.75$ & $6 / 6$ & N5 & 63 & M \\
\hline \multirow[t]{2}{*}{5} & Aphake & Aphake & Successful & & $7.7 /+17.00$ & $6 / 6$ & N5 & $7.7 /+16.00$ & $6 / 6$ & N5 & 52 & M \\
\hline & \multicolumn{12}{|c|}{ Aphakic/Phakic } \\
\hline 6 & Aphake & Accomm & Discontinued & Did not attend follow up & $7.9 /+16.00$ & $6 / 6$ & N5 & & & & 37 & M \\
\hline 7 & Aphake & Accomm & Discontinued & Poor quality of near vision & $7.9 /+11.75$ & $6 / 6$ & N5 & & & & 46 & M \\
\hline 8 & Cataract & Aphake & Discontinued & Poor quality of near vision & 8.1/plano & $6 / 36$ & & $8.3 /+17.50$ & $6 / 6$ & N5 & 36 & M \\
\hline 9 & Aphake & Presby & Discontinued & Poor near vision. Requires $+4 \mathrm{D}$ add & $7.9 /+14.00$ & $6 / 5$ & N6 & & & & 59 & M \\
\hline 10 & Aphake & Presby & Discontinued & Did not attend follow-up & $8.2 /+17.00$ & $6 / 6$ & N10 & $8.2 /+1.75$ & $6 / 6$ & N8 & 57 & $\mathrm{~F}$ \\
\hline \multirow[t]{2}{*}{11} & Aphake & Accomm & Successful & & $7.6 /+12.50$ & 6/9 & N8 & & 6/9 & & 35 & M \\
\hline & \multicolumn{12}{|c|}{ Pseudophakic/High Refractive Error } \\
\hline 12 & Pseudo & Aphake & Discontinued & Poor near vision. Requires $+4 \mathrm{D}$ add & $7.9 /+1.50$ & $6 / 12$ & N18 & $7.9 /+16.75$ & $6 / 6$ & N12 & 61 & $\mathrm{~F}$ \\
\hline 13 & Presby & Pseudo & Successful & & $7.9 /+8.50$ & $6 / 6$ & N5 & $7.9 /+0.50$ & 6/9 & N5 & 51 & M \\
\hline 14 & Pseudo & Aphake & Discontinued & Poor quality of near vision & $7.7 /+3.00$ & 6/9 & N8 & $7.6 /+16.25$ & $6 / 6$ & N8 & 48 & $\mathrm{~F}$ \\
\hline
\end{tabular}

examination of the anterior segment was carried out.

Contrast sensitivity measurements were performed at the end of the period of lens wear at the Department of Optometry and Visual Science of The City University. Sine wave gratings at spatial frequencies of 2.1 to 8.5 cycles per degree were generated on a computer monitor. The sine wave amplitude was varied, commencing below threshold and increasing slowly until detected. The value recorded was an average of the levels at which the grating was 'just visible' and 'just invisible.'

The diffractive lenses were tested monocularly and binocularly against equivalent single vision lenses. Distance and near vision were simulated using plus and minus spectacle lenses. The tests were repeated with the addition of a glare source. Results of eight runs under each set of conditions were averaged.

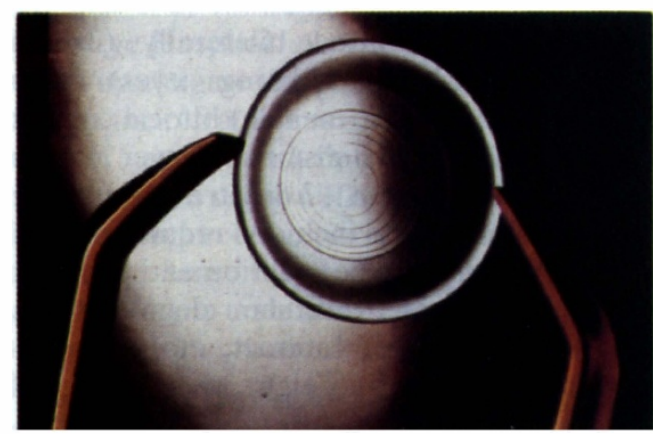

Fig 1. A gas permeable diffractive bifocal contact lens (+1 dioptre addition).
2. Sixteen pseudophakic patients fitted with Hema (soft) plano diffractive bifocal contact lenses were assessed for ease of visualisation of the fundus and retinal image quality using direct and indirect ophthalmoscopy, +90 dioptre and Hruby fundus lenses.

Goldmann peripheral fields were performed with and without diffractive lenses on four patients who had well centred implants and central, round pupils.

Informed consent was obtained from all patients taking part in these studies.

\section{Results:}

Six subjects completed the eight week period of contact lens wear without problems, were satisfied with the distance and near visual outcome and continued in their lenses.

In the Aphakic/Phakic category one patient was successful, two patients discontinued contact lens wear because of poor quality of near

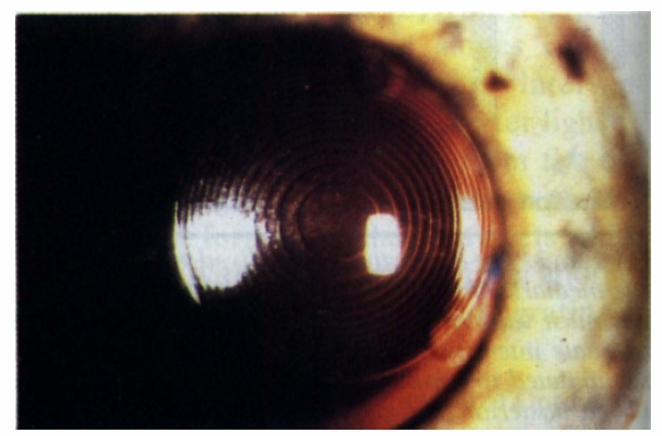

Fig 2. A soft diffractive bifocal contact lens in situ. 
vision. In one case this was due to an apparent requirement for an addition of +4 dioptres, and flare when driving. The remaining two patients in this category failed to attend further appointments after lenses were issued. Attempts to contact these patients were unsuccessful.

In the Aphakic/Aphakic category, four out of five patients were successful, while one discontinued due to epithelial problems.

In the Pseudophakic category, one patient complained of poor quality of near vision, another appeared to require a +4 dioptre addition for satisfactory near vision and the third was successful with the lenses provided.

Contrast sensitivity measurements, at the spatial frequencies tested, failed to reveal any reproducible difference between the diffractive and single vision lenses with either simulated distance or near vision.

Fundoscopy, using the direct and indirect ophthalmoscopes and the +90D and Hruby lenses, of sixteen pseudophakic patients wearing plano diffractive contact lenses revealed no reduction in the quality or area of the fundal view.

Goldmann perimetry was unaffected by diffractive lens wear.

\section{Conclusion}

Diffractive contact lenses may have a role in contact lens correction of aphakia, especially if bilateral, although they do not appear to provide a satisfactory compensation for the loss of accommodation in pseudophakic patients. Although one would expect a low degree of enthusiasm for contact lenses in patients with a low refractive error, even if the fellow eye is highly hypermetropic, it is significant that it was the quality of near vision that patients found unsatisfactory. Patients benefitting most from the lens were also noted to be those with lower reading requirements, although this was not formally assessed.

Lack of brightness of the near image was the most frequently stated problem. This is related to the inevitable optical contrast loss when a single image is split into two images and predictable from the ratio of $41.8 \%$ of light directed toward the near image and $41.8 \%$ for distance, that this lens produces. The remaining light is diffracted into other orders or scattered.
Contrast sensitivity measurements however, failed to show the predicted fall in contrast sensitivity when compared with single vision lenses, although the group tested was small, heterogeneous and only low spatial frequencies were examined. The step size used in the tests was in the region of $\sqrt{ } 2$. At low spatial frequencies the contrast loss of the diffractive lens may be less than this. Differences in contrast less than this step size are difficult to measure reliably but are none the less noticed by wearers.

Significantly two patients appeared to require a +4 dioptre reading addition when fitted, although their spectacle addition was measured at +3 dioptres. Four dioptre additions were not deemed practical for diffractive optic contact lenses, requiring twenty-four annular zones in the $5 \mathrm{~mm}$ diffractive area, and hence more light scatter. This may prove relevant to diffractive intraocular lenses.

The special cleaning regime used initially to protect the optical properties of the lens grooves proved to be unnecessary for the eight week trial period. Similarly, centration of the lens was not a limiting factor for near vision.

Although the diffractive optic provides no impediment to observation of the fundus, its effect on photocoagulation remains unproven.

Our results suggest that subjects for implantation of the diffractive lens must be carefully selected due to the high level of dissatisfaction with the quality of near vision.

The authors would like to thank Mr. Mark Heathcote of Moorfields Department of Medical Illustration and Mr. Christopher Hogg, also of Moorfields Eye Hospital, for their help with the illustrations.

Key Words: Aphakia, Bifocal Contact Lens, Diffractive Optic, Pseudophakia.

\section{References}

${ }^{1}$ Percival SPB: Prospective study of the new diffractive bifocal intraocular lens. Eye 1989; 3: 571-5.

${ }^{2}$ Freeman MH, Stone J. A new diffractive bifocal contact lens. Transactions of the British Contact Lens Association Annual Clinical Conference 1987; 4: 15-22.

${ }^{3}$ Charman WN: Diffractive bifocal contact lenses. Contax 1986; May: 11-17. 